\title{
Effectiveness of IL-23 Inhibitor Guselkumab in Real-World Chinese Patients with Psoriasis During a 20-Week Period
}

\author{
Yung Chan iD \\ Bik Sai Bessie Tong \\ Pui Yan Ngan \\ Chi Sum Au \\ Apex Dermatology Institute, Kowloon, \\ Hong Kong
}

Correspondence: Yung Chan

Room II 03-06, II/F Hong Kong Pacific

Centre, 28 Hankow Road, Tsim Sha Tsui,

Kowloon, Hong Kong

Tel + 85237033580

$\mathrm{Fax}+85237020018$

Email info@apexdermhk.com
Background: Interleukin-23 inhibitors are novel treatment options for psoriasis, and their efficacy and safety have been widely demonstrated in phase 3 clinical trials. Nonetheless, their real-world data remain limited, especially in Asia.

Objective: To evaluate the real-world effectiveness of interleukin-23 inhibitor guselkumab in Chinese patients with psoriasis.

Methods: In this retrospective single-center study, Chinese patients with psoriasis receiving a standard dose of guselkumab from November 2018 to May 2020 were included in the study cohort. Disease assessment was performed at baseline (Week 0), and at Week 4, 12, and 20 thereafter, using Psoriasis Area and Severity Index (PASI) score.

Results: Data of 68 adult patients with psoriasis were retrieved for analysis. At Week 20, $72.1 \% / 47.1 \%$ of the patients achieved PASI 90/100 response respectively, and $76.5 \%$ achieved a PASI score $<3$. Baseline mean PASI score was 17.5 , which significantly reduced to 2.0 at Week $20(\mathrm{P}=0.000)$. No previous use of biologics was a single significant factor associated with achieving PASI 90/100 and PASI score $<3$ responses at Week 20 (all $\mathrm{Ps}_{\mathrm{s}}<0.05$ ), while there were no statistically significant differences between males and females and body weight $>75$ and $\leq 75 \mathrm{~kg}$ in achieving these responses (all Ps $>0.05$ ). Adverse events were experienced by five patients (7.4\%), and all were mild in severity.

Conclusion: In this first real-world study on guselkumab among Chinese patients with psoriasis, this biologics was shown to be safe and effective in reaching an optimal clinical response up to 20 weeks.

Keywords: Chinese, guselkumab, interleukin-23, psoriasis, real-world

\section{Introduction}

Psoriasis is a chronic immune-mediated disease that primarily affecting the skin. ${ }^{1}$ Traditionally, clinical symptom control for patients with moderate-to-severe disease is achieved by phototherapy and systemic therapy such as cyclosporine, acitretin, and methotrexate. However, these conventional treatments may not be sufficiently effective and are associated with numerous side effects. ${ }^{2}$ In recent years, the advent of biological therapy provides new therapeutic options for psoriasis, including interleukin (IL)-12/23, IL-17, and IL-23 inhibitors. In particular, IL-23 is the latest cytokine discovered to play a crucial role in the pathophysiology of this skin disorder. It is regarded as a master regulatory cytokine in innate and adaptive immunity, as it initiates local chronic tissue inflammation in the upstream pathway (IL-23/T-helper Th17 cell axis) by activating a range of inflammatory cells such as 
Th17 cells. Subsequently, this causes the release of numerous downstream proinflammatory cytokines, eventually leading to keratinocyte activation and proliferation. ${ }^{3-5}$ These findings resulted in the development of IL-23 inhibitors as a novel class of biologics for the treatment of psoriasis.

Efficacy of biologics has been widely demonstrated in treating moderate-to-severe psoriasis. With the increasing availability of these novel agents with satisfactory clinical outcomes, a more aggressive treatment goal could be set aiming for almost clear/clear skin responses (ie absolute Psoriasis Area and Severity Index [PASI] $<3$ or PASI 100 response [complete remission]). Indeed, long-term randomized clinical trials indicated that percentage of patients with moderate-to-severe psoriasis achieving PASI 90 response (between 44 and 60 weeks) ranged from around 40-50\% (ustekinumab, adalimumab, and infliximab) to above $70 \%$ for the newer biologics (risankizumab, brodalumab, guselkumab, ixekizumab, and secukinumab). ${ }^{6}$ Recent studies demonstrated that half of the patients could achieve PASI 100 response receiving IL-23 inhibitors. ${ }^{7,8}$ These data render the latest American Academy of Dermatology National Psoriasis Foundation 2019 guidelines to recommend anti-IL biologic agents including IL-23 inhibitors as a monotherapy option in adult patients with moderate-tosevere plaque psoriasis. $^{9}$

Although randomized controlled trials are crucial in assessing drug efficacy and safety, the generated data are often different from those obtained in clinical daily practice. In view of this, we aim to assess the use of guselkumab, an IL-23 inhibitor approved in 2018 in Hong Kong, in treating local Chinese patients with psoriasis, in order to assess its real-world effectiveness and to corroborate the results of clinical trials.

\section{Materials and Methods Data Collection}

Data of adult patients with psoriasis were collected retrospectively at a private dermatology clinic in Hong Kong. The data retrieved for analysis included medical records on baseline patient demographics and previous biological treatments. Patients received guselkumab from November 2018 to May 2020 were recruited, and their treatment was initiated at different time during this period. Inclusion criteria of patients were age $\geq 18$ years with a clinical diagnosis of psoriasis who received treatment with guselkumab. Patients who had been treated with prior biological therapies (including tumor necrosis factor [TNF] inhibitors, IL-12/23 inhibitors, and IL-17 inhibitors) were also included, and no specific tapering off or washout period was noted for the previous treatments. Exclusion criteria included concomitant use of biologics other than guselkumab and those with incomplete patient record data.

All patients were given subcutaneous injection of guselkumab as a self-financed item at standard dosing, with an induction phase of $100 \mathrm{mg}$ administered at Week 0 , Week 4 , and a maintenance dose every 8 weeks thereafter. For every visit (Week 0, 4, 12, and 20), each patient was examined and his/her absolute PASI score was calculated in order to assess the clinical efficacy of guselkumab. Safety was evaluated at each follow-up visit according to adverse event profile. Patients with a follow-up period of at least 20 weeks were consecutively included in the analysis.

Ethical approval was obtained from the Hong Kong Human Research Independent Review Board. Patient consent was not required in this retrospective study because all the patient particulars were de-identified after study to ensure privacy and confidentiality of individual patient.

\section{Statistical Analysis}

Descriptive statistics were given as mean \pm standard deviation (SD) for continuous variables, and categorical variables were reported as numbers and percentages. Mean absolute PASI scores, PASI 90/100 response rates, and proportion of patients achieving a PASI score $<3$ were calculated at each time point.

One-way repeated measures ANOVA with post hoc Bonferroni test was used to detect differences in PASI scores among different time points. Fisher's exact test was used to assess the association of various factors including gender, body weight (dichotomized as $<$ or $>$ observed mean/median value), and previous biologic use with rates of PASI 90, PASI 100, or PASI score $<3$ at Week 4, 12 and 20. Statistics were done using IBM SPSS Statistics for Windows, Version 20.0. Armonk, NY: IBM Corp. Differences were considered statistically significant if two-sided $\mathrm{p}$ values $<0.05$.

\section{Results}

There were 68 patients included in the study for data analysis. All were Chinese and 60 of them (88\%) were diagnosed with moderate-to-severe psoriasis (PASI score $>10$ ) at baseline (Week 0). Mean age of overall patients was $41.2 \pm 12.4$ years, and mean body weight was $76.3 \pm 13.6 \mathrm{~kg}$. And, $27.9 \%$ of the patients had been given systemic oral 
conventional medication, $5.9 \%$ had been treated with phototherapy, $23.5 \%$ of the studied patients had been treated with traditional Chinese medicine. About two-thirds of patients ( $n=46 ; 67.6 \%$ ) were biologic-naïve at Week 0. For those who had been treated with previous biologics $(n=22)$, most received IL-17 inhibitors (secukinumab and ixekizumab). Twenty-two patients were previously treated with other biologics, $11(50 \%)$ of them switched to guselkumab due to loss of efficacy to the previous agents after 6-36 months (mean 18.5 months). One stopped secukinumab suffered from viral meningitis 13 months after commencement. Demographic and clinical baseline characteristics of patients are summarized in Table 1. The reasons for switching from previous biologics are shown in Table 2 .

All patients received guselkumab at standard dose. At Week 20,72.1\%/47.1\% of the patients achieved PASI 90/ 100 response, respectively, and $76.5 \%$ achieved a PASI score $<3$. At Week 0, mean PASI score was 17.5, and this value was significantly reduced to 2.0 at Week 20

Table I Baseline Patients' Characteristics ( $N=68)$

\begin{tabular}{|l|l|}
\hline $\begin{array}{l}\text { Gender } \\
\text { Male }\end{array}$ & $\mathbf{n}(\%)$ \\
$56(82.3)$ \\
\hline $\begin{array}{l}\text { Age } \\
\text { Mean (SD) } \\
\text { Median } \\
\text { Range }\end{array}$ & Years \\
\hline $\begin{array}{l}\text { Body weight } \\
\text { Mean (SD) } \\
\text { Median } \\
\text { Range }\end{array}$ & $41.2(12.4)$ \\
$18-77$
\end{tabular}

Note: ${ }^{+}$Previous medication may be more than one agent in each patient.

Abbreviations: SD, standard deviation; TNF, tumor necrosis factor; IL, interleukin.
Table 2 Reasons for Switching Biologics from Previous Biologics

\begin{tabular}{|l|l|}
\hline Reasons & $\mathbf{n}(\%)$ \\
\hline Primary inefficacy & $10(45.5)$ \\
Loss of efficacy & $1 \mathrm{I}(50)$ \\
Adverse events & $\mathrm{I}(4.5)$ \\
Total & $22(100)$ \\
\hline
\end{tabular}

Abbreviation: $\mathrm{n}$, number of patients.

$(\mathrm{P}=0.000)$ (Figure 1$)$. There was a statistically significant difference in mean PASI scores between the 4 time points (Week 0, 4, 12, and 20). Post hoc tests using the Bonferroni correction revealed that significant reduction in mean PASI scores was observed as early as Week 4 until Week 20 (all Ps=0.000 vs Week 0). Subgroup analysis indicated that for both biologic-naïve and biologicexperienced patients, initiating guselkumab resulted in a significant reduction in mean PASI scores, again as early as Week 4 until Week 20 (all Ps=0.000 vs Week 0) (Figure 1).

There were no statistically significant differences between males and females, as well as body weight $>75$ and $\leq 75 \mathrm{~kg}$ in achieving PASI 90/100 and PASI score $<3$ responses at Week 20 (all $\mathrm{Ps}_{\mathbf{0}}>0.05$ ), although PASI 90/100 rates appeared to be higher in female, older ( $>40$ years), and lighter $(\leq 75 \mathrm{~kg}$ ) patients. On the other hand, without the use of previous biologics was significantly associated with achieving PASI 90/100 and PASI score $<3$ responses at Week 20 (Table 3). Adverse events were reported in five patients, including allergic rhinitis, upper respiratory tract infection, urticaria, folliculitis, and injection site reaction, respectively, and all were considered mild in severity and easy to manage.

\section{Discussion}

To date, there are only limited post-marketing data available on the effectiveness and tolerance of guselkumab, ${ }^{10}$ especially in Asia where this agent is still novel which has been generally available later than the US. In this study, we retrospectively analyzed data of Chinese patients with moderateto-severe psoriasis treated with guselkumab in daily clinical practice. The observed PASI 90/100 response rates are in line with those from the international phase 3 VOYAGE1, 2 and ECLIPSE trials (at Week 16). ${ }^{11-13}$ According to a recent meta-analysis of pivotal clinical trials, PASI 90 rate was estimated at $72 \%$ and $39 \%$ for risankizumab and tildrakizumab respectively at 10-16 weeks, and PASI 100 rate at $40 \%$ and $14 \%$, respectively. ${ }^{6}$ Therefore, our PASI 90/100 results 


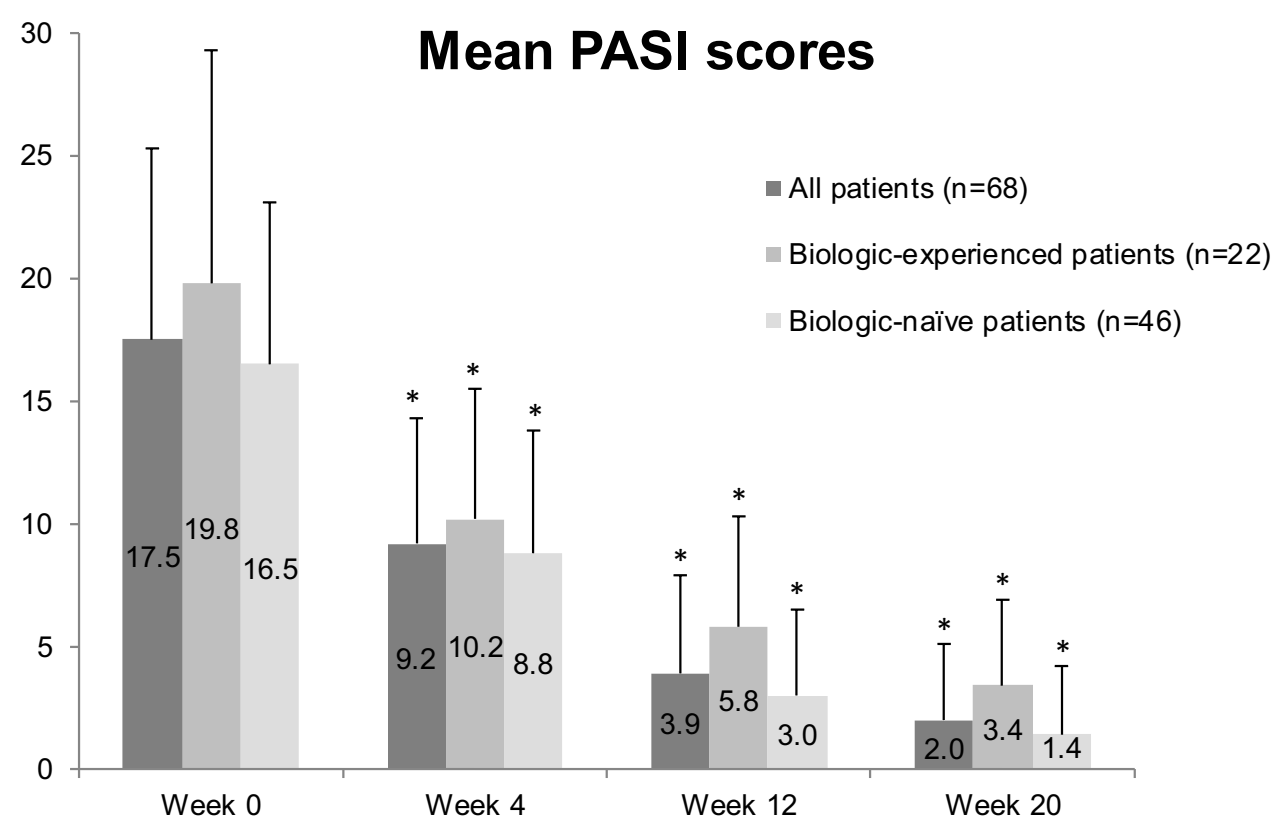

Figure I Mean PASI scores $( \pm S D)$ at different time points for all patients and patients stratified by prior biologic use. *All Ps $=0.000$ versus Week 0 for all groups, respectively.

(72.1\% and $47.1 \%$ at Week 20, respectively) appear to be on par with other IL-23 inhibitors, supporting the idea that guselkumab shows clinical benefits in real-world patients with moderate-to-severe psoriasis. Our results also demonstrated similar if not better efficacy to those obtained in another real-world retrospective data review conducted in

Table 3 Subgroup Analysis for PASI Response at Week 4, Week 12 and Week 20

\begin{tabular}{|c|c|c|c|c|c|c|c|}
\hline Subgroup & Time & PASI 90 & $P$ value & PASI I00 & $P$ value & PASI Score <3 & $P$ value \\
\hline $\begin{array}{l}\text { Female } \\
\text { Male }\end{array}$ & Week 4 & $\begin{array}{l}0 \% \\
3.6 \%\end{array}$ & 1.000 & $\begin{array}{l}0 \% \\
3.6 \%\end{array}$ & 1.000 & $\begin{array}{l}8.3 \% \\
5.4 \%\end{array}$ & 0.549 \\
\hline $\begin{array}{l}\text { Female } \\
\text { Male }\end{array}$ & Week 12 & $\begin{array}{l}25 \% \\
32.1 \%\end{array}$ & 0.742 & $\begin{array}{l}16.7 \% \\
21.4 \%\end{array}$ & 1.000 & $\begin{array}{l}58.3 \% \\
48.2 \%\end{array}$ & 0.752 \\
\hline $\begin{array}{l}\text { Female } \\
\text { Male }\end{array}$ & Week 20 & $\begin{array}{l}83.3 \% \\
69.6 \%\end{array}$ & 0.487 & $\begin{array}{l}58.3 \% \\
44.6 \%\end{array}$ & 0.527 & $\begin{array}{l}75.0 \% \\
76.8 \%\end{array}$ & 1.000 \\
\hline $\begin{array}{l}\text { Body weight } \leq 75 \mathrm{~kg} \\
\text { Body weight }>75 \mathrm{~kg}\end{array}$ & Week 4 & $\begin{array}{l}6.1 \% \\
0 \%\end{array}$ & 0.232 & $\begin{array}{l}6.1 \% \\
0 \%\end{array}$ & 0.232 & $\begin{array}{l}12.1 \% \\
0 \%\end{array}$ & 0.050 \\
\hline $\begin{array}{l}\text { Body weight } \leq 75 \mathrm{~kg} \\
\text { Body weight }>75 \mathrm{~kg}\end{array}$ & Week 12 & $\begin{array}{l}39.4 \% \\
22.9 \%\end{array}$ & 0.191 & $\begin{array}{l}24.2 \% \\
17.1 \%\end{array}$ & 0.555 & $\begin{array}{l}57.6 \% \\
42.9 \%\end{array}$ & 0.332 \\
\hline $\begin{array}{l}\text { Body weight } \leq 75 \mathrm{~kg} \\
\text { Body weight }>75 \mathrm{~kg}\end{array}$ & Week 20 & $\begin{array}{l}72.7 \% \\
71.4 \%\end{array}$ & 1.000 & $\begin{array}{l}51.5 \% \\
42.9 \%\end{array}$ & 0.627 & $\begin{array}{l}75.8 \% \\
77.1 \%\end{array}$ & 1.000 \\
\hline $\begin{array}{l}\text { No previous biologics } \\
\text { With previous biologics }\end{array}$ & Week 4 & $\begin{array}{l}2.2 \% \\
4.5 \%\end{array}$ & 0.546 & $\begin{array}{l}2.2 \% \\
4.5 \%\end{array}$ & 0.546 & $\begin{array}{l}6.5 \% \\
4.5 \%\end{array}$ & 1.000 \\
\hline $\begin{array}{l}\text { No previous biologics } \\
\text { With previous biologics }\end{array}$ & Week 12 & $\begin{array}{l}41.3 \% \\
9.1 \%\end{array}$ & 0.010 & $\begin{array}{l}28.3 \% \\
4.5 \%\end{array}$ & 0.027 & $\begin{array}{l}63.0 \% \\
22.7 \%\end{array}$ & 0.004 \\
\hline $\begin{array}{l}\text { No previous biologics } \\
\text { With previous biologics }\end{array}$ & Week 20 & $\begin{array}{l}80.4 \% \\
54.5 \%\end{array}$ & 0.042 & $\begin{array}{l}58.7 \% \\
22.7 \%\end{array}$ & 0.009 & $\begin{array}{l}89.1 \% \\
50.0 \%\end{array}$ & 0.001 \\
\hline
\end{tabular}

Abbreviation: PASI, psoriasis area and severity index. 
Italy, of which PASI 90, PASI 100 , and PASI $\leq 3$ response was achieved by $62.8 \%, 46.5 \%$, and $74.4 \%$ of the patients receiving guselkumab respectively at Week $20 .^{14}$

Data in the present study demonstrate gradual and significant reduction in absolute PASI scores through time. By Week $20,76.5 \%$ of the patients had a PASI score $<3$. It is noteworthy that absolute PASI has become a commonly used endpoint to evaluate therapeutic success in routine clinical practice. There is a growing consensus that PASI scores of $\leq 3$ may constitute better measures of therapeutic success. ${ }^{15}$ Achieving this feat by a great proportion of our patients is clinically meaningful as the ultimate goal of any psoriasis treatment is to achieve almost clear/clear skin, which has the additional benefit of improving patients' quality of life. In a psoriasis worldwide survey, $84 \%$ of all respondents experienced discrimination and/or humiliation due to psoriasis, and many reported negative effects on work, intimate relationships, sleep, and mental health. Patients without clear/almost clear skin reported that such achievement would open new possibilities, such as swimming (58\%), a wider choice of clothing (40\%), and meeting new people (26\%). ${ }^{16}$

It has been suggested that IL-23 inhibitors demonstrate a slower onset of action (eg as compared with IL-17 inhibitors). ${ }^{17}$ However, our results show that significant reduction in PASI scores was observed as early as Week 4 (the first time point for analysis). Indeed, the VOYAGE1 trial results suggested that the onset of action of guselkumab was even faster, with significant response observed as early as Week 2 compared with placebo. ${ }^{11}$ Nevertheless, more data may be needed to compare the onset of action of IL-23 inhibitors with that of previous IL inhibitors in clinical practice.

One of the values of real-world studies is to evaluate patients with elevated level of complexity, and therefore, our data set included those who switched from at least one class of biologics. In our study population, all but one biologic-experienced patients had moderate-tosevere psoriasis (PASI score $>10$, with a mean value of 19.8) at baseline and most received secukinumab previously. Switching to guselkumab was mainly due to secondary failure with prior biologics. Although treatment with guselkumab in this subgroup showed a subpar PASI 100 response rate at Week 20 (22.7\%), a significant reduction in mean PASI scores to $\sim 3$ was noted, and half of these patients achieved a PASI score $<3$ at Week 20. Indeed, relatively early loss of efficacy of secukinumab has been observed despite continuous treatment. ${ }^{18}$ In this case, switching to another biologic class with a different mechanism may be considered. ${ }^{19}$ Nevertheless, there has been a paucity of data on what to do next upon failure with a biologic..$^{19}$ Our data show much improved clinical outcomes in those who switched from previous biologics to guselkumab, and therefore providing an IL-23 inhibitor early may be considered for those who fail with other biologics. Nonetheless, the number of biologic-experienced patients in our sample is relatively small, and further studies may need to be conducted to specifically evaluate the potential of IL-23 inhibitors as a salvage therapy for this patient subgroup, or in a broader sense, to determine the treatment order of various classes of biologics for moderate-to-severe psoriasis. On the other hand, bio-naïve patients in this study were found to achieve higher PASI 90/100 and PASI score $<3$ responses, implying that guselkumab could have a potential role as an early biologic choice for patients, although whether it can serve as an effective long-term treatment option for real-world patients remains to be seen.

Treatment with guselkumab was well tolerated, with only a handful of patients reporting mild adverse events, including allergic rhinitis, upper respiratory tract infection, urticaria, folliculitis, and injection site reaction. All of the observed side effects were considered manageable, and did not cause any treatment discontinuation. Also, none of the patients reported inflammatory bowel disease and candidiasis, both have been noted in patients treated with IL-17 inhibitors. ${ }^{17,20,21}$

Limitations of the current study include the lack of long-term follow-up data being available. Although it has been reported in the updated VOYAGE1 trial results that guselkumab showed sustainable response up to 5 years, ${ }^{22}$ its long-term real-world use remains to be investigated. In addition, quality of life data are not collected, and therefore the impact of achieving almost clear/clear skin on patients' daily life cannot be assessed. Also, caution must be taken with our relatively small sample size, and further studies are needed to confirm our data by using a more representative and variable patient population. Nonetheless, to our understanding, this is the first study assessing the effectiveness of guselkumab in real-world Chinese patients with psoriasis. Our results confirm that this biologic demonstrates optimal short-term treatment outcomes with a good tolerability profile. 


\section{Funding}

The present study did not receive funding.

\section{Disclosure}

The authors declare no conflicts of interests.

\section{References}

1. Sawyer LM, Malottki K, Sabry-Grant C, et al. Assessing the relative efficacy of interleukin-17 and interleukin-23 targeted treatments for moderate-to-severe plaque psoriasis: a systematic review and network meta-analysis of PASI response. PLoS One. 2019;14(8): e0220868. doi:10.1371/journal.pone.0220868

2. Reich K, Mrowietz U. Treatment goals in psoriasis. J Dtsch Dermatol Ges. 2007;5(7):566-574.

3. Gaffen SL, Jain R, Garg AV, Cua DJ. The IL-23-IL-17 immune axis: from mechanisms to therapeutic testing. Nat Rev Immunol. 2014;14 (9):585-600. doi:10.1038/nri3707

4. Gooderham MJ, Papp KA, Lynde CW. Shifting the focus - the primary role of IL-23 in psoriasis and other inflammatory disorders. $J$ Eur Acad Dermatol Venereol. 2018;32(7):1111-1119.

5. Langrish CL, McKenzie BS, Wilson NJ, de Waal Malefyt R, Kastelein RA, Cua DJ. IL-12 and IL-23: master regulators of innate and adaptive immunity. Immunol Rev. 2004;202(1):96-105. doi:10.1111/j.0105-2896.2004.00214.x

6. Armstrong AW, Puig L, Joshi A, et al. Comparison of biologics and oral treatments for plaque psoriasis: a meta-analysis. JAMA Dermatol. 2020;156(3):258-269. doi:10.1001/jamadermatol.20 19.4029

7. Ferris LK, Ott E, Jiang J, et al. Efficacy and safety of guselkumab, administered with a novel patient-controlled injector (One-Press), for moderate-to-severe psoriasis: results from the phase 3 ORION study. $J$ Dermatolog Treat. 2020;31(2):152-159. doi:10.1080/ 09546634.2019.1587145

8. Yang EJ, Smith MP, Ly K, Bhutani T. Evaluating guselkumab: an anti-IL-23 antibody for the treatment of plaque psoriasis. Drug Des Devel Ther. 2019;13:1993-2000.

9. Menter A, Strober BE, Kaplan DH, et al. Joint AAD-NPF guidelines of care for the management and treatment of psoriasis with biologics. J Am Acad Dermatol. 2019;80(4):1029-1072.

10. Fougerousse AC, Ghislain PD, Reguiai Z, et al. Effectiveness and short-term (16-week) tolerance of guselkumab for psoriasis under real-life conditions: a retrospective multicenter study. $J$ EurAcad Dermatol Venereol. 2020;34(10):e644-e646.
11. Blauvelt A, Papp KA, Griffiths CEM, et al. Efficacy and safety of guselkumab, an anti-interleukin-23 monoclonal antibody, compared with adalimumab for the continuous treatment of patients with moderate to severe psoriasis: results from the Phase III, double-blinded, placebo- and active comparator-controlled VOYAGE 1 trial. $J \mathrm{Am}$ Acad Dermatol. 2017;76(3):405-417.

12. Reich K, Armstrong AW, Foley P, et al. Efficacy and safety of guselkumab, an anti-interleukin-23 monoclonal antibody, compared with adalimumab for the treatment of patients with moderate to severe psoriasis with randomized withdrawal and retreatment: results from the phase III, double-blind, placebo- and active comparator-controlled VOYAGE 2 trial. J Am Acad Dermatol. 2017;76(3):418-431.

13. Reich K, Armstrong AW, Langley RG, et al. Guselkumab versus secukinumab for the treatment of moderate-to-severe psoriasis (ECLIPSE): results from a phase 3, randomised controlled trial. Lancet. 2019;394(10201):831-839. doi:10.1016/S0140-6736(19) 31773-8

14. Galluzzo M, Tofani L, Lombardo P, et al. Use of guselkumab for the treatment of moderate-to-severe plaque psoriasis: a 1 year real-life study. J Clin Med. 2020;9(7):2170. doi:10.3390/jcm9072170

15. Del Alcázar Viladomiu E, Lamas Doménech N, Salleras Redonnet M. Absolute versus relative psoriasis area and severity index in clinical practice. Actas Dermo Sifiliogr. 2019;110(7):606-610.

16. Armstrong A, Jarvis S, Boehncke WH, et al. Patient perceptions of clear/almost clear skin in moderate-to-severe plaque psoriasis: results of the clear about psoriasis worldwide survey. J EurAcad Dermatol Venereol. 2018;32(12):2200-2207.

17. Costanzo A, Eyerich K. Discover the potential: exploring new frontiers of IL-23 inhibitors. Eur Med J. 2019;4(1):12-19.

18. Huang YYM, Ruth JS, Hsu S. Loss of efficacy of secukinumab for psoriasis at 24 to 32 weeks. $J$ Am Acad Dermatol. 2016;75(4):e169. doi:10.1016/j.jaad.2016.05.049

19. Krader CG. Guidance on transitioning patients between biologics. Dermatol Times. 2020;41(4):23.

20. Saunte DM, Mrowietz U, Puig L, Zachariae C. Candida infections in patients with psoriasis and psoriatic arthritis treated with interleukin-17 inhibitors and their practical management. $\mathrm{Br}$ J Dermatol. 2017;177(1):47-62. doi:10.1111/bjd.15015

21. Machado Á, Torres T. Guselkumab for the treatment of psoriasis. Bio Drugs. 2018;32(2):119-128. doi:10.1007/s40259-018-0265-6

22. Griffiths CEM, Papp KA, Song M et al. Maintenance of response through 5 years of continuous guselkumab treatment: results from the phase 3 VOYAGE 1 trial. Poster presented at 16th Annual Coastal Dermatology Symposium (Virtual); October 15-16; 2020.
Psoriasis: Targets and Therapy

\section{Publish your work in this journal}

Psoriasis: Targets and Therapy is international, peer-reviewed, open access journal focusing on psoriasis, nail psoriasis, psoriatic arthritis and related conditions, identification of therapeutic targets and the optimal use of integrated treatment interventions to achieve improved outcomes and quality of life. Visit http://www.dovepress. com/testimonials.php to read real quotes from published authors. 